\title{
Graubünden unterstützt Hausärzte in der Peripherie
}

Franz-Martin Janggen ${ }^{a}$, Martin Mani ${ }^{b}$

a Bündner Ärzteverein, verantwortlich für den Notfalldienst

b Kantonsarzt, Graubünden
Korrespondenz:

Dr. med. F. M. Janggen

Gäuggelistrasse 60

CH-7000 Chur

Tel. 0812523633

fjanggen[at]bluewin.ch
Die Belastung durch den ärztlichen Notfalldienst (NFD), der von Hausärzten in der Praxis erbracht wird, ist ein Dauerthema. Ein Hausärztemangel zeichnet sich vor allem in den wenig dicht besiedelten Gebieten des Kantons Graubünden ab. Betroffen sind auch Tourismusregionen, wo während der Hochsaison wenige Ärzte für eine grosse Anzahl Bewohner und Touristen bei gesundheitlichen Problemen bereitstehen müssen. In der Zwischensaison sind die Arztpraxen nur schlecht ausgelastet, die Dienstbelastung infolge Pikettdienst ist aber sehr gross und nicht kostendeckend. In diesem Umfeld sieht sich der Kanton Graubünden mit der Herausforderung konfrontiert, die frei werdenden Arztpraxen vor allem in Randregionen besetzen zu können. Eine Steigerung der Attraktivität und eine Verbesserung der Rahmenbedingungen sind unumgänglich, um diese Regionen für den Hausärztenachwuchs interessant zu machen.

Die Organisation des NFD ist auch im Kanton GR von der Regierung an den Bündner Ärzteverein (BüAeV) mittels Leistungsvereinbarung delegiert worden. Aufgrund der Topographie des Kantons sind in vielen Dienstarztregionen nur ein bis sechs Grundversorger tätig. Bei der bestehenden Altersstruktur der Praxisinhaber wird man in den nächsten Jahren für viele Praxen einen Nachfolger suchen müssen. Die Regionalspitäler wiederum sind oft weit entfernt. Nicht überall wird es möglich sein, alternative Formen wie Gruppenpraxen oder Praxen in Spitälern einzurichten. Die Verpflichtung zum Notfalldienst schreckt viele junge Allgemeinmediziner ab, in ein Gebiet mit wenigen Grundversorgern zu ziehen. Die sozialen Bedürfnisse der eigenen Familie und die Weiterbildungspflicht haben grössere Abwesenheiten zur Folge.

In Anbetracht dieser veränderten Rahmenbedingungen sah sich der BüAeV veranlasst, sein Reglement über den ärztlichen Notfalldienst zu überarbeiten; die neue Regelung trat am 1.1.2011 in Kraft. Die Kernpunkte sind folgende: Der BüAeV kann wegen fehlender personeller Ressourcen (Dienstärzte/DA) nicht mehr in allen Regionen einen flächendeckenden NFD über 365 Tage im Jahr gewährleisten. Weiter wird in diesem Reglement die Notfalldienstzeit der einzelnen Dienstärzte geregelt. In der Regel soll ein DA an Wochenend- oder Feiertagen zu nicht mehr als 12,5 Tagen NFD pro Jahr verpflichtet werden können. Müssen aufgrund der geringen Anzahl Dienstärzte in einer Region mehr Diensttage (an Wochenenden und Feiertagen) geleistet werden, müssen diese vergütet werden. Zudem muss die Möglichkeit für mindestens 7 Wochen Abwesenheit (Ferien, Weiterbildung) pro Jahr bestehen.
Die Bündner Regierung hat die Zeichen der Zeit erkannt und zusammen mit dem BüAeV Lösungsvorschläge ausgearbeitet, die zu verbesserten Rahmenbedingungen führen sollen. Bei der Revision des Krankenpflegegesetzes (KPG) hat der Grosse Rat des Kantons Graubünden die notwendigen rechtlichen Grundlagen geschaffen, um die Tätigkeit der DA grundsätzlich abgelten zu lassen (Art. 36 Abs. 1 KPG). Der Beitrag des Kantons (90\%) und der Gemeinden (10\%) für die Funktion der DA beträgt gemäss Budget für das Jahr 2012770000 Franken. Gemäss Art. 31b Abs. 1 VOzKPG sind Ärzte und Ärztinnen für die über die 12,5 Diensttage an Wochenenden und Feiertagen pro Jahr hinausgehenden Diensttage mit 800 Franken pro Tag zu entschädigen. Dieser Betrag wird aufgrund der gesetzlichen Regelung an die entsprechenden Regionalspitäler ausbezahlt und auf die DA aufgeteilt. Sofern einzelne Regionalspitäler die DA entlasten, z.B. indem sie an Wochenenden den Notfalldienst übernehmen und auch Hausbesuche ausführen (hausärztlicher NFD), bleibt der entsprechende Betrag beim Regionalspital.

Mit dieser Lösung ergibt sich für die mit vielen Notfalldiensten belasteten Ärzte also die Möglichkeit, sich zu entlasten, sei dies durch Zusammenarbeit mit dem Regionalspital, die Anstellung eines Praxisvertreters oder andere Formen.

Die Dienstpläne werden seit 2012 im ganzen Kanton einheitlich im System docbox erstellt. Dadurch ist es auf einfache Art möglich, das notwendige Zahlenmaterial für die Auszahlungen zu berechnen. Zusätzlich zu der nun eingeführten, oben beschriebenen Dienstarztentschädigung unterstützt der Kanton Graubünden nachhaltig die ärztliche Grundversorgung im Kanton:

- Die Regierung unterstützt den ärztlichen Notfalldienst der frei praktizierenden Ärzte durch Beiträge an die Ausbildung in der Höhe von 2000 Franken für den Grundkurs bzw. 500 Franken für den Refresherkurs sowie für die Dienstarztausrüstung in der Höhe von jährlich 2000 Franken pro Arzt.

- Das Projekt «Capricorn» hat das Ziel, für angehende Grundversorger Ausbildungsstellen in Praxen zu schaffen und so den Nachwuchs für die Hausarztmedizin in unserem Kanton zu gewinnen. Um den Bestand der heute existierenden Grundversorgerpraxen nach Möglichkeit zu sichern, werden vier Ausbildungsplätze für Assistenzärzte für je ein halbes Jahr durch den Kanton mitfinanziert. Das Programm war praktisch vom ersten Tag an ständig ausgebucht. 